2. Considering $\mathrm{rSO}_{2}$ more depends on circulatory sufficiency then $\mathrm{FiO}_{2}$ it is expedient to use such a definition as 'oxygenous price of saturation'. In that case using of Doppler estimation of cerebral blood flow pattern allows differing mixed hypoxia and cerebral ischemia aiming to choose proper ways of intensive care.

\section{PO-0478 MULTI-ORGAN DYSFUNCTION SCORING IN NEONATAL ENCEPHALOPATHY}

${ }^{1}$ DU Sweetman, ${ }^{2}$ JFA Murphy, ${ }^{3} \mathrm{~V}$ Donoghue, ${ }^{4} \mathrm{R}$ Segurado, ${ }^{2} \mathrm{EJ}$ Molloy. ${ }^{1}$ Neonatology, National Maternity Hospital and National Children's Research Centre, Dublin, Ireland; ${ }^{2}$ Neonatology, National Maternity Hospital, Dublin, Ireland; ${ }^{3}$ Radiology, National Maternity Hospital, Dublin, Ireland; ${ }^{4}$ Biostatistics - CSTAR School of Public Health and Population Science, University College Dublin, Dublin, Ireland

10.1136/archdischild-2014-307384.1119

Background Neonatal encephalopathy secondary to perinatal asphyxia in term infants commonly results in multi-organ dysfunction (MOD). However methods to accurately and objectively quantify the degree of MOD in NE are lacking.

Objective To develop a scoring system which accurately reflects the degree of MOD in NE and to assess the ability of this score to predict outcome.

Methods Eighty-five term infants with NE were recruited. A score was assigned to abnormalities in each organ system ( $\mathrm{n}=$ 6) with a maximum score of 15 . E.g. In the cardiovascular system, Troponin-T $>0.1 \mathrm{ng} / \mathrm{ml}=1, \mathrm{HR}<80=1$. The higher the score assigned, the more significant the MOD. Scores were compared with outcomes of grade of encephalopathy/mortality.

Results Eighty infants had data for all the scoring variables and were included. Higher MOD scores were significantly associated with grade II/III NE (p-value <0.001) and with mortality ( $\mathrm{p}<$ $0.001)$ although numbers were small $(\mathrm{n}=4)$. ROC analysis showed that the MOD score was highly predictive of grade II/III NE with AUROC curve $=0.94(p<0.001)$ and cut-off value for best prediction of this outcome was 4.5. For prediction of mortality, AUROC curve was $0.96(p=0.002)$ and cut-off value was 10.5 .

Conclusion A scoring system that accurately reflects the degree of MOD in NE would be helpful and allow more objective assessment and comparison of these infants. The proposed MOD score needs to be prospectively validated in term newborns with NE. However it appears that our MOD score in NE is highly predictive of grade II/III NE and mortality.

\section{PO-0479 EARLY SCREENING FOR AUTISM SPECTRUM DISORDERS (ASD) IN PRETERM INFANTS: CORRELATION WITH PERINATAL DATA AND PREDICTIVE VALUES}

${ }^{1} \mathrm{~V}$ Tenorio, ${ }^{2} \mathrm{~A}$ Mancera, ${ }^{1} \mathrm{~F}$ Botet, ${ }^{1} \mathrm{JM}$ Rodriguez-Miguelez, 'D Salvia, ${ }^{1} \mathrm{~J}$ Figueras-Aloy. ${ }^{1}$ Neonatal Department, ICGON. BC Natal. Agrupació Sanitària Hospital Clínic - Hospital Sant Joan de Déu. IDIBAPS. Facultat de Medicina. Universitat de Barcelona. Barcelona., Barcelona, Spain; ${ }^{2}$ Psycology Department, Universitat Autonoma de Barcelona, Barcelona, Spain

\subsection{6/archdischild-2014-307384.1120}

Background Prematurity increases the rate of ASD. Therefore preterm infants should be screened for it. Screening tests are designed for 24 months onwards. However, some traits of ASD are present at an earlier age and early intervention seems to improve prognosis.
Aims (1) Study the prevalence of ASD at 12 and 24 months postmenstrual age. (2) Compare results at both ages. (3) Explore correlation with perinatal data.

Methods Infants born $<33$ weeks. At 12 months, parents completed a questionnaire (CSBS-DP), which measures three areas: Communication, Expressive Speech and Symbolic abilities. At 24 months they completed the CSBS-DP and another questionnaire (M-CHAT). Results were compared at both ages and were correlated with neonatal morbidity data.

Results Correctly questionnaires were obtained in 121 infants at $12 \mathrm{~m}$ and 43 infants at $24 \mathrm{~m}$. Mean gestational age (GA) was 29.31 weeks (SD 2.39). At $12 \mathrm{~m}, 19 \%$ infants had abnormal total scores (Communication 15.7\%, Speech 25\%, Symbolic 20.7\%). At $24 \mathrm{~m}, 16.7 \%$ had abnormal scores (Communication $23.8 \%$, Speech $21.4 \%$, Symbolic $14.3 \%$ ), and $24.3 \%$ abnormal M-CHAT. Rate change from 12 to $24 \mathrm{~m}$ was significant for Communication ( $\mathrm{p}=0.006$ ). PPV ranged $0.81-0.91$ and NPV $0.31-0.60$. Abnormal results correlated with umbilical artery $\mathrm{pH}$ $<7.15$ ( $\mathrm{p}=0.034)$, Apgar scores $<7$ at $5 \mathrm{~min}(\mathrm{p}=0.035)$, twin pregnancy $(p=0.049)$, abnormal brain scans $(p=0.005)$, surgery $(p=0.027)$ and a trend with male gender and lower GA. Conclusions The CSBS-CP questionnaire correlates with neonatal morbidity. It shows a high PPV although a variable NPV when compared at $24 \mathrm{~m}$. It seems a good early screening, although a close follow-up is always needed.

\section{PO-0480 PRENATAL EVALUATION AND POSTNATAL EARLY OUTCOMES OF FETAL VENTRICULOMAGALY}

${ }^{1} \mathrm{~A}$ Tugcu, ${ }^{2} \mathrm{C}$ Gulumser, ${ }^{1} \mathrm{~A}$ Abbasoglu, ${ }^{2} \mathrm{~N}$ Uysal, ${ }^{1} \mathrm{~A}$ Ecevit, ${ }^{3} \mathrm{E}$ Kupana, ${ }^{2} \mathrm{~F}$ Bilgin Yanik, ${ }^{1} \mathrm{~A}$ Tarcan. 'Pediatrics Neonatology, Baskent University, Ankara, Turkey; ${ }^{2}$ Obstetrics and Gynecology Perinatology, Baskent University, Ankara, Turkey; ${ }^{3}$ Pathology, Baskent University, Ankara, Turkey

\subsection{6/archdischild-2014-307384.1121}

Objective This study aims to determine the incidence, aetiology, diagnostic criteria and early outcomes of prenatally diagnosed fetal ventriculomegaly (VM).

Methods Diagnostic criteria for the fetal VM was atrial diameter of lateral ventricle measuring $\geq 10 \mathrm{~mm}$, independent from gestational age. Results of our patients from ultrasonography (USG), karyotyping, congenital infections, and associated abnormalities were noted. Progress during pregnancy, postnatal USG results and outcomes after birth were recorded.

Results In our study, 40 cases of fetal VM were recorded. Female to male fetus ratio was (19/21) 0,90. Median gestational age at detection of VM was 22 weeks (ranging between 16-34 weeks). Sixteen of the $40 \mathrm{VM}$ cases were bilateral (40\%), 24 of the $40 \mathrm{VM}$ cases were unilateral (60\%). Twenty-one VM cases were isolated $(52.5 \%)$, there were associated structural abnormalities in 19 of the $40 \mathrm{VM}$ cases $(47.5 \%)$. One case was complicated by toxoplasmosis $(1 / 40)(2.5 \%)$. Nineteen of the $40 \mathrm{VM}$ cases had amniocentesis $(47,5 \%)$ and 2 of them showed abnormalities (10.5\%). Twenty four of the $40 \mathrm{VM}$ cases were improved and returned to normal during pregnancy (60\%). Eight pregnancies were terminated (8/40) 20\%). Three babies passed away during neoanatal and postneonatal period. Six babies classified in our mild "isolated" VM class were found to have some structural abnormalities after birth.

Conclusions Our report revelaed that associated abnormality incidence and termination rate among mild VM cases were high. Although most of mild VM cases are thought to be benign, 
associated abnormalities should be carefully evaluated and determined pre- and postnatally.

\section{P0-0481 CEREBRAL MRI VERSUS COMBINED TERM/NEONATAL ULTRASONOGRAPHY FOR PRETERM LESIONS DIAGNOSIS}

${ }^{1} \mathrm{P}$ Vo Van, ${ }^{1} \mathrm{G}$ Loron, ${ }^{1} \mathrm{H}$ Meunier, ${ }^{2} \mathrm{G}$ Mac, ${ }^{1} \mathrm{P}$ Morville, ${ }^{1} \mathrm{~N}$ Bednarek. ${ }^{1}$ Neonatology, Institut Alix de Champagne, Reims, France; ${ }^{2}$ Radiology, Institut Alix de Champagne, Reims, France

\subsection{6/archdischild-2014-307384.1122}

Background/aims Brain magnetic resonance imaging (MRI) is the reference technique for the assessment of qualitative injuries and cerebral growth. However cerebral ultrasonography (CUS) remains the standard follow-up imaging for most neonatologists. The aim of the study is to compare term corrected age MRI to a protocol combining term and neonatal CUS (tCUS/nCUS).

Methods Between January 2013 and March 2014, preterm (PT) $\leq 30$ gestational weeks $(\mathrm{GW})$ received the same day term corrected age MRI and tCUS in addition to classic nCUS. Supratentorial (intraventricular haemorrhage (IVH), nodular and cystic periventricular leukomalacia $(\mathrm{PVL})$ ) and cerebellar lesions were

Abstract P0-0481 Table 1 Ultrasound sensivity (Se) and specificity for qualitative lesions compared to MRI according to the tie of investigation

\begin{tabular}{|c|c|c|c|c|c|c|c|c|}
\hline & $\begin{array}{l}\text { Mild }(n= \\
\text { Severe IV } \\
(n=5)\end{array}$ & $\begin{array}{l}\text { 24)/ } \\
\text { H }\end{array}$ & $\begin{array}{l}\text { Nodu } \\
(\mathrm{n}=\mathrm{S}\end{array}$ & & \multicolumn{2}{|c|}{$\begin{array}{l}\text { Cystic PVL } \\
(\mathrm{n}=2)\end{array}$} & \multicolumn{2}{|c|}{$\begin{array}{l}\text { Cerebellar } \\
\text { lesions } \\
(n=9)\end{array}$} \\
\hline & Se $(\%)$ & Sp (\%) & $\mathrm{Se}$ & $\mathrm{Sp}$ & $\mathrm{Se}$ & $\mathrm{Sp}$ & Se & Sp \\
\hline nCUS & $62.5 / 100$ & $100 / 97,9$ & 0 & 100 & 50 & 100 & & \\
\hline tCUS & $62.5 / 80$ & $100 / 100$ & 11.11 & 100 & 100 & 99 & 0 & 100 \\
\hline tCUS+nCUS & $100 / 100$ & $97.4 / 95,9$ & 11.11 & 100 & 100 & 99 & & \\
\hline
\end{tabular}

assessed separately. Corpus callosum length (CCL), a myelination marker, was measured on MRI and tCUS. Sensitivity (Se) and specificity ( $\mathrm{Sp}$ ) were calculated for qualitative lesions, metrics were compared by linear regression.

Results The study included prospectively 102 PT. Mean GW and birth weight were $26.9( \pm 2.2)$ and $1127 \mathrm{~g}( \pm 313)$. Table described the results. Linear regression coefficient for CCL was 0.7 .

Conclusion Additional tCUS raises diagnosis rate of mild IVH and cystic PVL and is reliable for CCL measures. However MRI remains superior for nodular white matter and cerebellar lesions.

\section{PO-0482 SECRETONEURIN AS A POTENTIAL DIAGNOSTIC MARKER OF NEONATAL HYPOXIC-ISCHAEMIC ENCEPHALOPATHY}

${ }^{1} \mathrm{~K}$ Wechselberger, ${ }^{1} \mathrm{M}$ Höck, ${ }^{1} \mathrm{~A}$ Posod, ${ }^{1} \mathrm{~A}$ Schmid, ${ }^{1} \mathrm{M}$ Urbanek, ${ }^{2} \mathrm{R}$ Fischer-Colbrie, ${ }^{1} \mathrm{U}$ Kiechl-Kohlendorfer, ${ }^{1} \mathrm{E}$ Griesmaier. ${ }^{1}$ Department of Pediatrics II (Neonatology), Innsbruck Medical University, Innsbruck, Austria; ${ }^{2}$ Department of Pharmacology, Innsbruck Medical University, Innsbruck, Austria

\subsection{6/archdischild-2014-307384.1123}

Background Hypoxic-ischaemic encephalopathy (HIE) is a major cause of neurologic impairment and mortality in neonates suffering from perinatal asphyxia. Early information on prognosis is of great importance to guide therapeutic decisions and to inform the parents. The neuropeptide secretoneurin (SN) was shown to be a promising early serum biomarker of an unfavourable neurological outcome in adult patients after cardiopulmonary resuscitation. Currently, there are no studies on SN levels in neonates available.

Aim To determine physiological SN levels in healthy term neonates and to assess its potential as a biomarker for HIE in newborn infants.

Methods At present this study prospectively enrolled 46 healthy term born neonates ( 29 male) and four term born neonates with moderate to severe HIE. SN levels were assessed by radioimmunoassay in cord blood.

Results In healthy newborn infants mean SN serum levels in cord blood were $126.33 \pm 87.47 \mathrm{fmol} / \mathrm{ml}$. In the four patients diagnosed with moderate to severe HIE, SN levels in cord blood were significantly higher compared to healthy controls $(253.48$ $\pm 102.55 \mathrm{fmol} / \mathrm{ml}, \mathrm{p}<0.05)$.

Conclusion SN serum levels in healthy neonates contained on average $126 \mathrm{fmol} / \mathrm{ml}$, which is in accordance with elevated SN serum levels observed in children compared to adults in a previous study. In patients with moderate to severe HIE SN serum levels were significantly increased compared to healthy controls. We are currently enrolling further patients to investigate a possible association with the neurological outcome.

\section{PO-0483 PROSPECTIVE OBSERVATIONAL STUDY OF DOXAPRAM FOR THE TREATMENT OF APNEA OF PREMATURITY}

${ }^{1} \mathrm{~J}$ Winter, ${ }^{1} \mathrm{C}$ Bratek, ${ }^{1} \mathrm{~B}$ Fekker, ${ }^{1} \mathrm{~A}$ Kidszun, ${ }^{2} \mathrm{~S}$ Schmidtmann, ${ }^{1} \mathrm{C}$ Whybra, ${ }^{1} \mathrm{E}$ Mildenberger. 'Department of Neonatology, Children's Hospital of the University Medical Center of the Johannes Gutenberg University Mainz, Mainz, Germany; ${ }^{2}$ Institute of Medical Biometry Epidemiology and Informatics, University Medical Center of the Johannes Gutenberg University Mainz, Mainz, Germany

\subsection{6/archdischild-2014-307384.1124}

Background and aims After the implementation of a local treatment protocol at our NICU, we aimed to systematically evaluate if intubation for apnea of prematurity was avoided by doxapram. We asked, if frequency and severity of apneas were affected and if side effects occurred.

Methods We prospectively analysed all premature infants $<30$ weeks treated according to a standardised protocol during 10/ 2010 to $04 / 2013$. Doxapram was given only, if otherwise intubation had been necessary. We registered the number of apneas, bradycardias, and desaturations, $\mathrm{pCO}_{2}$ and side effects an hour before, at the start of, and during $48 \mathrm{~h}$ after onset of treatment. Results 21 of $66(31.8 \%)$ infants (mean gestational age 25.5 weeks, mean birth weight $705 \mathrm{~g}$ ) were treated during $2 \frac{1}{2} 2$ years. All of them had been treated with caffeine and CPAP before doxapram was applied. In 13 of 67 (19\%) therapy courses, infants were intubated because of persistent apnea during $48 \mathrm{~h}$ of doxapram treatment. The frequency of apneas (2.7 vs. 0.2), bradycardias $<80 / \mathrm{min}$ (1.0 vs. 0.2 ), and desaturations $<80 \%(3.4$ vs. 1.1) per hour decreased. Therapy was ceased in 5 cases because of side effects (gastrointestinal disturbances, seizure, extreme myoclonia).

Conclusions Intubation was avoided in a large proportion of cases. In addition, frequency and severity of apneas diminished. Thus, doxapram seems to be effective. However, in face of side effects and lack of long term outcome data, it should be used 